Journal of Neurology, Neurosurgery, and Psychiatry, 1980, 43, 369-371

Short report

\title{
A double blind study of metoclopramide in the treatment of migraine attacks
}

\author{
P TFELT-HANSEN, J OLESEN, A AEBELHOLT-KRABBE, \\ B MELGAARD, AND B VEILIS \\ From the Department of Neuromedicine, Rigshospitalet, Copenhagen, Denmark
}

SUMMARY One hundred and fifty patients with migraine attacks attending the Copenhagen acute migraine clinic were treated either with metoclopramide $10 \mathrm{mg}$ i.m., metoclopramide $20 \mathrm{mg}$ as suppository or placebo in a double blind trial. All patients simultaneously or 30 minutes later received paracetamol $1 \mathrm{~g}$ and diazepam $5 \mathrm{mg}$ orally. The nausea was relieved in $71 \%$ of the patients by placebo and bed rest, but metoclopramide was significantly $(p=0.04)$ more effective and relieved nausea in $86 \%$ of the patients. Metoclopramide did not by itself reduce the pain, but enhanced the effect of the analgesic or sedative medication. This effect, however, just failed to be statistically significant $(\mathrm{p}=0.06)$

Nausea, vomiting and delayed absorption of analgesics due to gastric stasis are usual features of the acute migraine attack. Phenothiazine-like drugs have most often been used as antiemetics, but they have anticholinergic effects, which could counteract gastric emptying. ${ }^{1}$ It has been shown that metoclopramide is an effective antiemetic. ${ }^{2}$ At the same time it promotes emptying of the stomach and normalises the absorption of aspirin during migraine attacks. ${ }^{3}{ }^{4}$ Based on this twofold rationale metoclopramide has been introduced as a supplement to analgesics in the treatment of the acute migraine attack. ${ }^{5}$ We have conducted a double blind trial with metoclopramide in 150 patients suffering from acute migraine attacks. The study was conducted in an acute migraine clinic, where the patients could be observed throughout the attack. Our aim was to evaluate the effect on nausea and to see if metoclopramide increased the effectiveness of the analgesic-sedative treatment with paracetamol and diazepam.

\section{Patients and methods}

The trial involved 150 patients with classical or

Address for reprint requests: Dr $P$ Tfelt-Hansen, Department of Neuromedicine, Rigshospitalet, DK 2400, Copenhagen, Denmark.

Azzepted 22 January 1980 common migraine as defined by the Ad Hoc Committee on Classification of Headache. ${ }^{6}$ They further fulfilled the criteria suggested by Olesen. ${ }^{7}$ The patients presented themselves with a migraine attack at the acute migraine clinic in Copenhagen. The organisation as well as diagnostic and therapeutic principles of this clinic have previously been described. ${ }^{8}$ Patients are treated in the clinic for individual acute attacks. Patients with marked nausea or vomiting were consecutively selected. There were 17 men and 133 women. The median age was 40.5 years (range 18-74 years). The duration of the migraine was more than 15 years in $60 \%$ of the patients. The median frequency of attacks was 1.5 per month. The actual attack for which the patient was treated had lasted for less than 4 hours in $12 \%$, from $4-24$ hours in $63 \%$ and from 25 hours and upwards in $25 \%$ of the cases.

After a brief standardised history and neurological examination, headache and nausea were graded from 0 to $3(0=$ none, $1=$ mild, $2=$ moderate and $3=$ severe). Nausea was severe in $63 \%$, moderate in $29 \%$ and mild in $8 \%$ of the cases. Vomiting in the preceding two hours was recorded in $27 \%$. Headache was severe in $71 \%$, moderate in $28 \%$ and mild in $1 \%$. The patients went to bed in a quiet, cool and dark room. They randomly received either placebo intramuscularly+ placebo supposi- 
tory (51 patients), metoclopramide $10 \mathrm{mg}$ intramuscularly and placebo suppository (49 patients), or placebo intramuscularly and metoclopramide suppository $20 \mathrm{mg}$ (50 patients). As analgesic and sedative treatment all patients were given paracetamol (aceteminophen) $1 \mathrm{~g}$ and diazepam $5 \mathrm{mg}$ orally. For $60 \%$ of the patients this was postponed 20 to 30 minutes because of severe nausea or vomiting. At the time of postponed treatment, pain and nausea were rated again. The patients were then left alone, but supervised quietly by a nurse. If they required further treatment, metoclopramide as injection or oral analgesics were allowed after 1 hour, and the patients were rated again at this time. Finally the patients were rated when they left the clinic and were asked to indicate the exact time at which the nausea had disappeared.

After the trial the data were evaluated blindly. An excellent result of the nausea treatment was defined as a decrease on the rating scale for nausea of 2 steps or to 0 within one hour. An excellent result for the analgesic-sedative treatment was defined as a decrease on the rating scale for headache of 2 steps or to 0 , no further treatment and a stay in the clinic of less than 8 hours.

\section{Results}

Because of violation of the protocol, ten and 14 patients were excluded blindly from the evaluation of nausea and analgesic-sedative treatment, respectively.

To test the comparability of patients used for evaluation of the treatment results, the following factors in the three treatment groups were compared: age, sex, duration of disease, prophylactic treatment, attacks per year, interictal headache, duration of actual attacks, number of vomitings, rating on nausea-scale and rating on headache-scale. No significant differences were found ( $p$ for all factors greater than $0 \cdot 10$, Chi-square test).

The results of the trial are shown in the table. Metoclopramide relieved the nausea in $86 \%$ whereas placebo was successful in $71 \%$ of the patients. In the metoclopramide group better results of the analgesic-sedative treatment were seen than in the placebo group, but the difference did not reach significance. The median length of stay in the clinic was 7 hours in both groups. No side effects were noted.

To evaluate the effect of metoclopramide on migraine pain, we examined the pain scores of patients with postponed analgesic-sedative treatment. Fifty-six patients had an excellent result of the nausea treatment rated before receiving the analgesicsedative treatment. The pain scores in these patients
Table Metoclopramide in the treatment of migraine attacks

\begin{tabular}{|c|c|c|c|c|}
\hline & \multicolumn{2}{|c|}{ Nausea treatment } & \multicolumn{2}{|c|}{ Analgesic-sedative treatment } \\
\hline & Excellent* & Unsatisfactory* & Excellent* & Unsatisfactory* \\
\hline \multirow{4}{*}{$\begin{array}{l}\text { Placebo } \\
\text { Metocloprami } \\
\text { i.m. } \\
\text { Metocloprami } \\
\text { supposi- }\end{array}$} & $\begin{array}{r}35 \\
\text { ide }\end{array}$ & 14 & 18 & 29 \\
\hline & $\begin{array}{r}37 \\
\text { ide }\end{array}$ & 5 & 19 & 21 \\
\hline & 41 & 8 & 29 & 20 \\
\hline & \multicolumn{2}{|c|}{$p=0.04 \dagger$} & \multicolumn{2}{|c|}{$\mathrm{p}=0.06+$} \\
\hline
\end{tabular}

"Defined in "Patients and Methods".

†Fischer's exact test, one tail. For the statistical calculation both metoclopramide groups were considered together.

showed a decrease of one step in 16 patients (seven received placebo) and of two steps in three patients (one received placebo). Pain scores were unchanged in 33 patients.

\section{Discussion}

The present study is the first controlled trial of metoclopramide used in combination with a mild analgesic agent against migraine attacks. Despite the marked placebo effect it could be shown that metoclopramide was statistically significantly better than placebo. Significance was, however, only obtained by considering the injection group and the suppository group together, and by using a one tail statistical test. It has been found that intramuscular administration of metoclopramide is twice as effective as oral administration in increasing the threshold of apomorphine-induced vomiting in man. ${ }^{\ominus}$ Since the suppositories used in the present study contained twice as much metoclopramide as given intramuscularly, it should be acceptable to consider the two groups together, Metoclopramide is known to be a highly effective antiemetic agent in a number of other conditions. ${ }^{2}$ We therefore thought it justified to disregard the possibility that it would worsen the nausea of migraine and consequently accepted a one-tail statistical test.

In previous clinical trials metoclopramide was given orally to migraine patients either daily for 3 months ${ }^{10}$ or together with ergotamine in individual attacks. ${ }^{11}$ Both studies showed that metoclopramide was significantly superior to placebo and thus support the findings of the present study.

The absorption of acetylsalicylic acid during a migraine attack correlates inversely to the severity of nausea, but may be normalised by metoclopramide. ${ }^{42}$ By this mechanism metoclopramide could be expected to improve the effect of oral analgesics. Our data indicate that this is so, but the results just failed to be statistically significant. The difference in pain response was seen in patients with 
poor nausea relief whereas the pain was ameliorated to the same extent in patients with good nausea relief irrespective of whether they had received metoclopramide or placebo. Therefore, placeboinduced relief of nausea may also have improved the absorption.

The most surprising result of the present investigation and a very interesting one was the very high placebo effect on nausea $(71 \%)$. The placebo response was not due to diazepam, since the nausea disappeared or greatly diminished in $66 \%$ of 31 patients before they received anything other than placebo. The placebo reactors were not statistically significantly different from the rest of the patients, when a number of clinical parameters were compared.

Generally, nausea and vomiting have been regarded as less influenced by psychological factors than pain in the migraine attack. It now seems that the reverse is true. For the majority of patients in this study, as treatment with placebo or active medication took effect, a stage was reached where nausea and other accompanying symptoms had disappeared, but the headache persisted. Later the headache diminished and disappeared. This is the reverse order of the development of symptoms at the start of an attack of common migraine.

We thank Lundbeck \& Co, Copenhagen, for supplying the test medication.

\section{References}

1 Wainscott G, Kaspi T, Volans. The influence of thiethylperazine on the absorption of effervescent aspirin in migraine. Br J Clin Pharmacol 1976; 3:1015-21.

2 Pinder RM, Brogden LN, Sawyer PR, Speight TM, Avery GS. Metoclopramide: A review of its pharmacological properties and clinical use. Drugs 1976; 12:81-131.

3 Kreel L. The use of metoclopramide in radiology. Postgrad Med J (Suppl 4) 1973; 42-6.

4 Volans GN. The effect of metoclopramide on the absorption of effervescent aspirin in migraine. $\mathrm{Br} J$ Clin Pharmacol 1975; 2:57-63.

5 Volans GN. The treatment of migraine. In: Turner P ed. Advanced medicine. Topics in therapeutics. Vol. 2 London: Pitman Medical, 1976: 156-72.

6 Ad hoc committee on classification of headache of the NIH. Classification of headache. JAMA 1962; 179:717-8.

7 Olesen J. Some clinical features of the acute migraine attack. An analysis of 750 patents. Headache 1978; 18:268-71.

8 Olesen J, Aebelholt A, Veilis B. The Copenhagen acute headache clinic. Organisation, patient material and treatment results. Headache 1979; 19:223-7.

9 Klein RL, Militello TE, Ballinger CM. Antiemetic effect of metoclopramide. Evaluation in humans. Anesth Analg 1976; 44:259-64.

10 Matts SGF. Metoclopramide in the treatment of migraine. Practitioner 1974; 212:887-90.

11 Slettnes O, Sjaastad O. Metoclopramide during attacks of migraine. In: Sicuteri $\mathrm{F}$ ed. Headache: New Vistas. Florence: Biomedical Press 1977: 201-4.

12 Volans GN. Absorption of effervescent aspirin during migraine. $\mathrm{Br}$ Med $J$ 1974; 4:265-9. 